\title{
DSCHAMI UND MAHALLE DES TOJGUN PASCHA IM STADTTEIL BUDA-VÍZIVÁROS
}

\author{
GYŐZŐ GERŐ* \\ (Budapest)
}

\begin{abstract}
Die Topographie des türkenzeitlichen Buda (Ofen) wurde von Lajos Fekete auf Grund der Quellen so exakt bestimmt, daß die archäologischen Freilegungen deren Richtigkeit in nahezu vollem Umfang bestätigt haben. Eines der am frühesten entstandenen türkischen Stadtviertel bzw. Befestigungsabschnitte Ofens war der Mahalle des Tojgun Pascha. Das Zentrum dieses Stadtteils bildeten die von Tojgun Pascha gestifteten Gebäude. Zu dem Gebäudekomplex Tojgun Paschas gehörten, wie Ewlija Tschelebi schreibt, eine Dschami, ein Doppelbad - Tschifte Hamam genannt -, eine Medresse und auch Läden. Letztere mögen in einem kleineren Bedesten- oder Arasta-Gebäude untergebracht gewesen sein, das mit dem auf dem Stadtplan von de la Vigne in der Nähe der Dschami dargestellten Bau mit Säulenhalle identisch sein dürfte. Von den Stiftungseinrichtungen Tojgun Paschas ging allein die 1553-1555 erbaute Dschami unbeschädigt aus der Belagerung des Jahres 1686 hervor; in der Kapuzinerkirche, der heutigen unterwasserstädtischen Pfarrkirche, blieben bedeutende Details der Dschami erhalten. Die bei der Freilegung der Kibla-Mauer »in situ« zum Vorschein gelangten Fenster bzw. die im Kircheninneren entdeckten Reste der Mihrab, des türkenzeitlichen Fußbodenniveaus und Kanzelfundaments (Mimber) haben es ermöglicht, den Grundriß des Gebäudes sowie seine südöstliche Fassade authentisch zu rekonstruieren.

Im Zuge der dortigen Grabungen kam auch ein kleines Detail des zwischen 1555 und 1556 errichteten, dann aber vollständig zerstörten Bades zutage, auf Grund dessen zweifelsfrei festgestellt werden konnte, daß dies ein Dampfbad gewesen ist.
\end{abstract}

Stichwörter: Architektur, Baukunst, Befestigungsabschnitt, Stadtteil des Tojgun Pascha, Archäologische Forschung, Bauforschung, Ansicht, Vorstadt, Stiftung, Gebäudeverzeichnis, Doppelbad.

Mit dem Anspruch nahezu vollkommener Genauigkeit bestimmte als erster Lajos Fekete in seinem die Feststellungen betreffend bis heute verläßlichen Werk die Topographie der türkenzeitlichen Stadt Buda (Ofen). ${ }^{1}$ Große Sorgfalt widmete er dabei neben den bedeutenderen Gebäuden auch der Bestimmung der Lage und Ausdehnung der einzelnen Stadtteile, der Mahallen. Besonders gewissenhaft untersuchte er die topographischen Angaben der ehemaligen ungarischen bzw. türkischen Stadt unter dem Aspekt der Kontinuität sowie des Erscheinens der einzelnen Gebäude und

* Győző Gerő, H-1014 Budapest, Tárnok u. 5, Hungary.

${ }^{1}$ Fekete (1944, pp. 72-122). 
Stadtteile auf dem Stadtplan der Gegenwart. Auf der Grundlage seiner Arbeit kann man den historischen Stadtgrundriß Ofens vom Mittelalter über die Türkenzeit bis in unsere Tage verfolgen.

Fekete schuf mit Hilfe der reichlich verfügbaren türkischen und westlichen Quellen einen Situationsplan der türkischen Stadt Ofen, den spätere archäologische Forschungen höchstens präzisieren konnten, ohne ihn jedoch grundlegend zu verändern.

In den letzten Jahrzehnten, wie auch in jüngster Vergangenheit, kam es in dem Stadtteil Víziváros (Wasserstadt) zu bedeutenden Ausgrabungen und Forschungen, die nicht nur im Hinblick auf das Mittelalter, sondern auch die Türkenzeit zahlreiche neue Ergebnisse brachten, unter anderem was die genauen topographischen Beobachtungen betrifft. ${ }^{2}$ Eines der verschiedenen - im Zentrum einzelner mittelalterlicher Stadtbezirke - erforschten Gebiete der türkischen Víziváros war der Mahalle des Tojgun Pascha. Hier bot sich neben archäologischen Freilegungen auch die Möglichkeit, Bauforschungen zu betreiben.

Der Mahalle Tojgun Paschas im Stadtteil Víziváros war einer der am frühesten entstandenen Mahallen des türkischen Ofen. Ewlija Tschelebi beschreibt ihn in seinem Bericht über die »Große Vorstadt«, d.h. die »Wasserstadt«, folgendermaßen: »Durch das Ahor-Tor eintretend muß man sich nach Westen wenden und diese Stadtteile werden Festungsabschnitt des Dujgun Pascha genannt. Er hat eine eigene Burgmauer. Durch dessen Tor eintretend führt die Hauptstraße [die heutige Fö utca] wieder in westliche Richtung, und diese Stadtteile nennt man Festungsabschnitt des Suleiman Pascha. ${ }^{3}$ Fekete bestimmt den Bölme Tojgun Paschas im heutigen Stadtgebiet wie folgt: »Vom Burgberg konnte man in der Gegend der heutigen Apor-Gasse über die zur Donau führende Bölmemauer hinaus durch das in dieser befindliche Istálló-Tor (Ahorluk-Tor) in den Bölme des Tojgun Pascha gelangen, der sich ungefähr bis zur Redoute erstreckte. An diesen grenzte im Norden der Bölme Suleiman Paschas. $\ll^{4}$ Wie im Folgenden zu sehen sein wird, modifizieren die neueren Forschungen letztere Feststellung nur insofern, als das Gebäude der ehemaligen Ofner Redoute noch im Gebiet des Bölme bzw. Mahalle lag. ${ }^{5}$ Allem Anschein nach waren das Gebiet des Bölme und des Mahalle gleichen Namens tatsächlich identisch, nachdem dieses teilweise von Mauern begrenzt wurde. Auch Ewlija Tschelebi bezeichnet den Mahalle des Tojgun Pascha als einen der namhafteren Stadtbezirke.

Tojgun Pascha bekleidete im Anfangszeitraum der Türkenherrschaft zweimal das Amt des Ofner Paschas. ${ }^{6}$ Wie seine Vorgänger bzw. Nachfolger war auch er bestrebt, die Stadt durch von ihm gestiftete Bauten zu bereichern, damit den Grundstein für das Zentrum des seinen Namen tragenden Stadtbezirks legend. Unter seinen Stiftungen in Ofen erwähnt Ewlija Tschelebi die Dschami und Medresse sowie das Bad

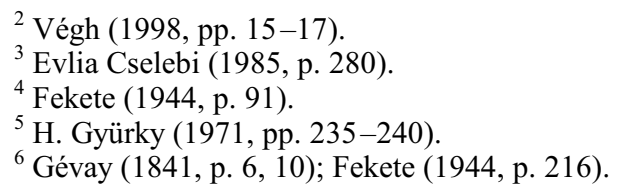


des Tojgun Pascha. ${ }^{7}$ Fekete markiert die Stelle der Stiftungseinrichtungen Tojgun Paschas »in der Gegend der heutigen Kapuzinerkirche«, wo - wie er sagt - »wahrhaft nur die Stiftungen von Mustafa Pascha und Tojgun Pascha eine rein türkisch-mohammedanische Atmosphäre um sich verbreiteten «.

Die großangelegte Bautätigkeit Tojgun Paschas ist vermutlich in die erste längere Zeitspanne seines Aufenthaltes in Ofen zu setzen. Als erster berichtet Hans Dernschwam darüber, der Anfang August des Jahres 1555 auf der Durchreise in Ofen weilt und auch der Víziváros einen Besuch abstattet. Er schreibt: »Zw Offen lest der jeczig Thoigan bascha ein gewaldig badt pawen, alda vor das augustiner kloster, schwarcze munich gewesen, dohin er von allen ortten die schonisten stainen furen lest. $\ll^{9}$ Dernschwam kannte Ofen noch aus der Zeit vor den Türken und von mehreren Besuchen recht gut, so daß man seinem Bericht in topographischer Hinsicht Glauben schenken darf, wie es auch die jüngsten Grabungen belegen. Albert Gárdonyi meinte früher irrtümlich, das mittelalterliche Augustinerkloster habe sich in der Nähe des Szombat-Tores befunden. ${ }^{10}$ Die Beobachtung von Fekete, wonach es im südlichen Teil der heutigen Fö utca zu suchen ist, kommt der Wahrheit näher. Diesbezüglich bestätigen die Beobachtungen der archäologischen Forschung die Authentizität der Mitteilung Dernschwams in verschiedener Hinsicht.

Nach dem Bericht von Dernschwam waren die Bauarbeiten am Bad 1555 noch im Gange, was mit großer Wahrscheinlichkeit voraussetzt, daß die das Zentrum des Mahalle bildende Dschami damals schon gestanden haben dürfte. Im folgenden möchte ich in erster Linie die Forschungen im Zusammenhang mit der Dschami des Tojgun Pascha sowie deren Ergebnisse besprechen und versuchen, unter Verwendung der Analogien eine hypothetische Rekonstruktion zu erstellen.

Vermutungen zufolge schloß das heutige Gebäude der Kapuzinerkirche (Unterstädtische Pfarrkirche) auf dem Grundstück Fö utca Nr. 32 bedeutende Details der Dschami Tojgun Paschas ein, was auch die vor Beginn der Forschungen an Ort und Stelle durchgeführten Untersuchungen zu bekräftigen schienen.

Die Dschami Tojgun Paschas erscheint während der Zeit der Türkenherrschaft und auch nach der Rückeroberung mehrfach in den Schriftquellen. Einen sicheren Anhaltspunkt in bezug auf die Dschami und die übrigen Gebäude bieten die Ende des 17. Jahrhunderts von Kriegsingenieuren angefertigten Aufnahmen. Und zwar insbesondere der Ofen darstellende Situationsplan von de la Vigne, ${ }^{11}$ ihn kann man als den authentischsten betrachten (Abb. 1). Er markiert nämlich nicht nur die Dschami, sondern auch das Bad, ein größeres Gebäude mit Säulenhalle - vermutlich eine Verkaufshalle - sowie dessen am nördlichen, östlichen und westlichen Ende mit jeweils einem Rundturm bewährte Mauer. Letztere dürfte mit großer Wahrscheinlichkeit die nördliche Abschlußmauer des Bölme gewesen sein.

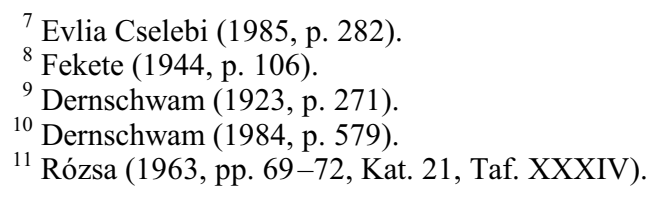


Marsigli kennzeichnet in seinem türkischen Stadtplan ${ }^{12}$ nur die Dschami, doch im Verzeichnis der türkischen Bauten von Ofen führt er auch das Bad an. ${ }^{13}$

Ein 1782, nach der Rückeroberung, angefertigter Situationsplan von der Dschami, die sich damals schon im Besitz der Kapuziner befand, und den umliegenden Gebäuden zeigt trotz verschiedener Umbauten gut die Grundrißanordnung der Dschami. $^{14}$

Wie bereits erwähnt, berichtet Dernschwam nur vom Bau des Bades. Kaum zwei Jahrzehnte später, im Jahr 1578, stattet Lubenau der Víziváros einen Besuch ab und beschreibt deren Dschami recht ausführlich, leider ohne ihren Namen zu verzeichnen. Dennoch geht aus der ziemlich detaillierten Beschreibung eindeutig hervor, daß sie sich auf die Dschami Tojgun Paschas bezieht. Lubenau erinnert sich: »Den 21. Februárii seindt wir mit den Janicsaren ausgangen, die Stadt zu besehen, und erstlich in der untern Stadt am Wasser zu einer schonen Moskea komen, welche mit Blei bedeckt. Daneben ein hoher Thurm und ein Gang herumb, auf welchem die turkische Pfaffen alle drey Stunden Tag und Nacht zum Gebett ruffen, weil sie gahr keine Glocken haben. Imwendig hingen viel gleserne Lampen, und stunden zwei groser messings Leuchter; der Boden wahr mitt schönen Tepichen bedeckt und wahr ein hoher, steinerner Predigstul drein; in dieser Moskea leidt der Bassa begraben. $\ll^{15}$

Von den schweren Kämpfen bei der Rückeroberung Ofens 1686 ist die Dscha$m i$ größtenteils verschont geblieben, so daß die kaiserlichen Truppen sie schon während der Belagerung in Gebrauch nehmen konnten. Hier sowie in anderen Gebäuden der Vakuf Tojgun Paschas brachte man die zum Christentum übergetretenen Gefangenen unter.

Die einzige topographisch authentische Grundrißdarstellung der Vakuf-Gebäude des Tojgun Pascha-Mahalle hat die Karte der Ofner Belagerung des Jahres 1686 von de la Vigne überliefert. Die Karte gibt das gesamte Platz- und Straßennetz der Víziváros wieder, das in dem Zustand bis Ende des 19. Jahrhunderts nahezu unverändert blieb und selbst auf dem heutigen Stadtgrundriß noch gut zu verfolgen ist. Die zur Karte gehörende Legende erwähnt in der Víziváros - der Unterstadt - sieben Moscheen und zwei Bäder, allerdings ohne Angabe der Namen. Moscheen markiert sie mit dem Zeichen $» Z \ll$, Bäder mit dem Zeichen $» \& «^{16}{ }^{16}$ Der Grundriß beider Gebäudetypen ist schematisch: die Dschami stellt sie als einen an die Ecke eines Quadrats anschließenden kleinen Kreis, das Bad dagegen als ein kuppelgedecktes Rechteck dar. Diese Situation entspricht, auf den heutigen Stadtplan projiziert, dem gegenwärtig von der Fö utca - Ponty utca - Szalag utca begrenzten Gebiet (Abb. 2). Den Corvin tér (Corvin-Platz) natürlich eingeschlossen, an dessen nördlichem Ende - teilweise an der Stelle des Gebäudes der ehemaligen Ofner Redoute - de la Vigne auf seiner Karte den Grundriß eines großen rechteckigen Bauwerkes mit Säulenhalle und mit einem großen, runden Turm an der Nordwestecke markierte. Südlich von dem Turm,

\footnotetext{
${ }^{12}$ Veress (1906, nach Blatt 142).

${ }^{13}$ Veress (1906, p. 137, unter Nr. 9).

${ }^{14}$ Veremund (1927, Illustration).

${ }^{15}$ Sahm (1912-1920, I, p. 80).

${ }^{16}$ Rózsa (1963, p. 71).
} 


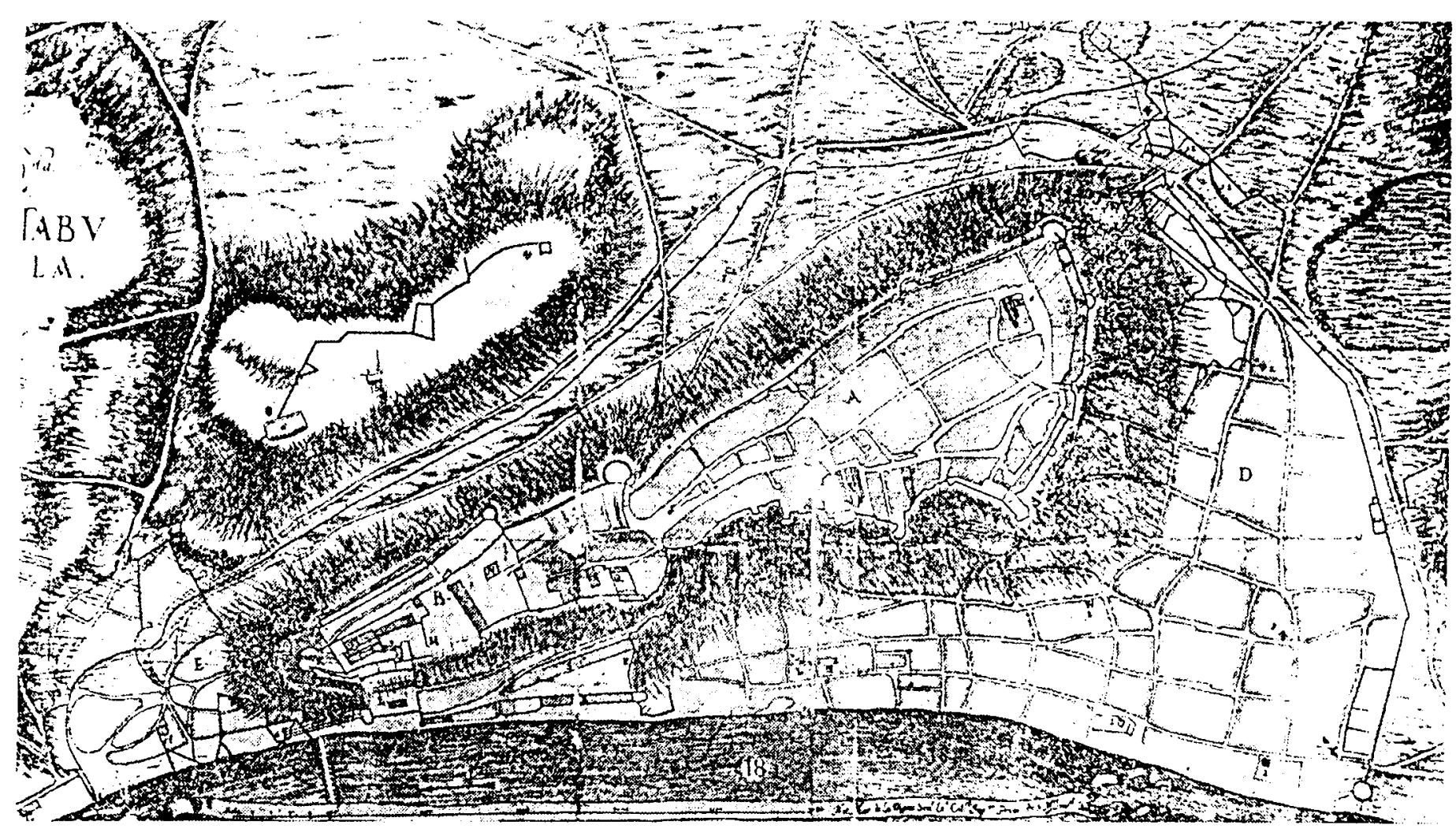

Abb. 1. Gebäudekomplex des Mahalle Tojgun Paschas auf der Ofen darstellenden Karte von de la Vigne, 1686 
auf der Ostseite der heutigen Fö utca, erhebt sich ein ähnlicher Turm, den eine Mauer mit dem vorigen verbindet. Dies dürfte die nördliche Abschlußmauer des Bölme gewesen sein. Um aber auf den Bau mit Säulenhalle zurückzukommen: $\mathrm{Ob}$ er eine Stiftung Tojgun Paschas war, weiß man nicht. Innerhalb des Mahalle jedoch muß es ein - in erster Linie für den Handel - ziemlich wichtiges Gebäude, vielleicht Arasta oder Bedesten usw., gewesen sein. Wie die hier durchgeführten Forschungen geringfügigeren Umfanges ergaben, geht es auf mittelalterliche Fundamente zurück. ${ }^{17}$

Die verschiedenen Darstellungen des Dschami-Gebäudes sind nicht als authentisch zu betrachten. Sämtliche erhalten gebliebenen Ansichten zeigen die Dschami schematisch, als würfelförmiges Bauwerk mit halbkugelförmiger Kuppel, an dessen nordwestlicher Ecke sich das Minarett mit umlaufendem Balkon erhebt. Dessen ungeachtet darf man aber auch jene Ansichten nicht außer acht lassen, die in der die Darstellung ergänzenden Legende eindeutig die Dschami Tojgun Paschas benennen. Und zwar an erster Stelle der die Ofner Belagerung des Jahres 1686 darstellende Stich von Magliar, wo in der Legende bei der mit Nr. 33 bezifferten Dschami »Toj Kun gyamesi. Tempio appellato del Vecello« zu lesen ist. ${ }^{18}$ Diese Bezeichnung kommt in verschiedenen Varianten auch auf anderen Stichen vor: »33. Ein kirch, von Vogel Toihun auff Türkisch genandt. ${ }^{19}$

Außer den bereits erwähnten Angaben finden sich sowohl in den türkischen, als auch in westlichen Schriftquellen so gut wie keine Hinweise, die die Dschami Tojgun Paschas oder seine anderen Stiftungseinrichtungen betreffen. Wieviel Personal in der Dschami Dienst versah und was es verdiente weiß man lediglich aus dem Jahr 1567. Laut Defter bestand das Personal damals aus acht Personen. Der monatlichen Zahlungsliste des Schaban zufolge hatte der Hatib das höchste »Gehalt« - 20 Aktsche pro Tag -, während die beiden Devrhan pro Kopf täglich bloß zwei Aktsche verdienten.

Ein Jahr später, 1568, findet man im Defter nur einen Hinweis auf den Stadtteil Turgut (Tojgun) Paschas, und zwar in Verbindung mit dem Nachlaß einer verstorbenen Frau. ${ }^{21}$ Am 20. September 1573 erwähnt eine Eintragung in den Ofner Rechnungsbüchern die von Tojgun Pascha im Mahalle gleichen Namens gegründeten Läden. Demnach hat ein Kürschner namens Jovan »in den Läden des Tojgun Pascha« für 1 Denk Pelzwerk 50 Aktsche Steuer bezahlt. ${ }^{22}$ Die genannten Läden befanden sich gewiß in dem oben beschriebenen Bauwerk mit Säulenhalle, dessen Funktion als Handelsstätte somit erwiesen scheint.

Mit weiteren Angaben über die Stiftungen und den Mahalle Tojgun Paschas dient erst wesentlich später wiederum Ewlija Tschelebi, als er 1664 Ofen beschreibt. In der ziemlich ausführlichen, auf die einzelnen Ofner Stadtteile - so die Víziváros -

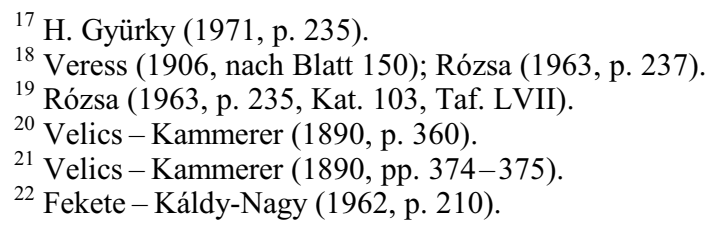




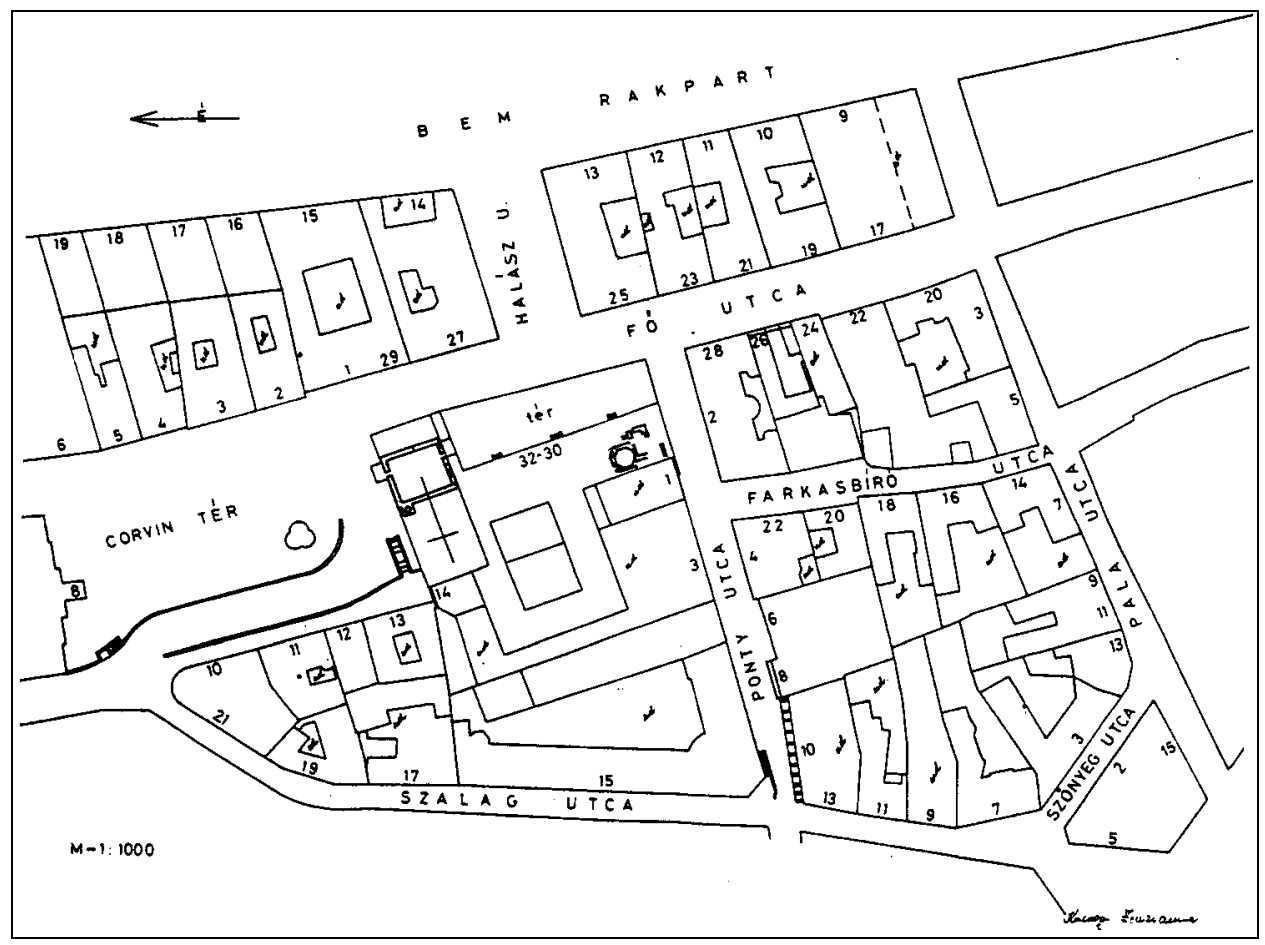

Abb. 2. Situationsplan des Corvin-Platzes und seiner Umgebung mit den freigelegten Resten der Dschami und des Bades Tojgun Paschas (Zsuzsanna Kuczoghi)

bezogenen Beschreibung schildert er auch den Bölme sehr anschaulich. Bei der Aufzählung der Mahallen erwähnt er den Stadtteil des Tojgun Pascha unter den berühmteren. Als Sehenswürdigkeiten der »Großen Vorstadt« beschreibt er vier »schöne und kunstvolle« Dschamis mit Bleidach, weiters fünf Medressen, wobei er die Medresse Tojgun Paschas namentlich nennt, ebenso wie bei der Schilderung der Bäder das Bad des Tujgun Pascha. ${ }^{23}$ Leider hebt Ewlija Tschelebi unter den Stiftungen Tojgun Paschas ausgerechnet dessen Dschami nicht namentlich hervor, und auch die dazugehörigen Läden erwähnt er mit keinem Wort. Die Medresse Mustafa und Tojgun Paschas dagegen kommt noch in mehreren späteren Quellen vor. ${ }^{24}$

Auf den Stadtansichten und Stadtplänen mit Darstellungen der erfolglosen Belagerung Ofens von 1684 und der zwei Jahre später - 1686 - geglückten Rückeroberung begegnet man die Dschami des Tojgun Pascha mehrfach und mit verschiedenen Variationen ihres Namens an. Das Bad erwähnt in erster Linie Marsigli, der es in seinem Ofner Gebäudeverzeichnis auch ausführlich beschreibt. ${ }^{25}$

${ }^{23}$ Evlia Cselebi (1985, p. 282).

${ }^{24}$ Fekete (1944, p. 290).

${ }^{25}$ Veress (1906, p. 137). Marsiglis Beschreibung deutet auf ein Doppelbad hin, was auch Zenarolla bekräftigt, wenn er sagt, daß die eine Hälfte von den Männern, die andere Hälfte von den Frauen benützt wurde. 
1687 übergab man die Dschami und die übrigen von Tojgun Pascha gestifteten Gebäude den Kapuzinern. Während der Belagerung wurden hier die zum Christentum bekehrten Gefangenen untergebracht. ${ }^{26}$

Im Zusammenhang mit den Restaurierungsarbeiten an der Kapuzinerkirche in Víziváros bot sich zweimal die Gelegenheit, die Überreste der Dschami zu erforschen, teilweise freizulegen und zu präsentieren. ${ }^{27}$ Bereits vor den Forschungen war auf Grund der Stadtpläne und Darstellungen des 17.-18. Jahrhunderts festzustellen, daß auch die Dschami Tojgun Paschas den Dschami-Typ mit Viereckgrundriß und einem Minarett vertrat, dem sich auf der Nordwestseite eine aus drei Gewölbefeldern bestehende, mit kleinen Kuppeln gedeckte Vorhalle anschloß. Bei den Forschungen konnte eindeutig geklärt werden, daß die Dschami ein bis zu den Grundmauern türkisches Bauwerk ist, das nicht, wie man früher vermutete, auf mittelalterliche Vorläufer zurückgeht.

Im Laufe des 18. Jahrhunderts kam es zu einer Erweiterung der heutigen Kapuzinerkirche, wobei die beiden Längsmauern der Dschami abgerissen wurden, so daß nur zwei ihrer Mauern stehen blieben. Davon war die Kibla-Mauer aus schön bearbeiteten, kaum sichtbar aneinander gefügten Sandsteinquadern und die nordwestliche Mauer aus Bruchsteinen aufgeführt. Die Abweichung zwischen den beiden Steinmauern resultiert meines Erachtens daraus, daß die nordwestliche Seite der Dschami während einer der Belagerungen - höchstwahrscheinlich der des Jahres 1602 - schweren Schaden erlitt und daher später neu aufgebaut werden mußte, wobei Material und Ausführung bereits minderer Qualität waren. Übrigens wurde diese Mauer der Dschami auch beim Umbau zur Kirche stark beschädigt, was zur fast völligen Vernichtung der türkischen Kunstformen führte, die nur noch in Spuren beobachtet werden konnten. Hier, an der Nordwestfront - auf deren Achse ursprünglich der Eingang zur Dschami lag -, zeigten sich Spuren einer ähnlichen Fenstereinteilung wie an der Südwestfassade, allerdings schon mit aus Ziegeln gestalteten Bögen und Fensterrahmenbett. Ebenso fand man im Obergeschoß dieser Gebäudefront zugemauerte Fensteröffnungen.

Am erfolgreichsten erwies sich Forschung an der südöstlichen Fassadenmauer der Dschami sowie im gegenwärtigen Kirchenschiff, in dem schmalen Abschnitt vor der Kibla-Mauer, und natürlich an der Innenfront der die Reste der Mihrab bergenden südöstlichen Hauptmauer. Darüber hinaus diente eine kleinere Grabung, die neben den archäologischen und Bauforschungen durchgeführt wurde, mit zahlreichen neuen Angaben über die Dschami.

Bei der Freilegung der Südostfassade stellte sich heraus, daß es eine homogene Quadermauer ist, in die man später - vermutlich im Laufe des 18. Jahrhunderts - ein Gewölbe gemeißelt hatte, und in der Neuzeit wurde die Mauer an mehreren Stellen mehr oder weniger stark beschädigt.

Die drei an diesem Fassadenabschnitt der Dschami entdeckten ursprünglichen Fensteröffnungen waren mit in Kalkmörtel gebetteten Bruchsteinen zugemauert. Da-

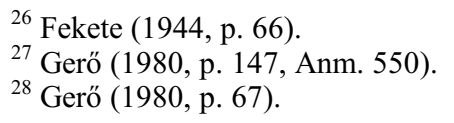


von konnte in ihrer ganzen Tiefe nur die Fensteröffnung des Erdgeschosses, und dann das unversehrt erhalten gebliebene Fenster selbst, freigelegt werden. Darüber, auf der Achse des Erdgeschossfensters, befand sich die Fensteröffnung des ersten Obergeschosses, deren oberen Abschluß man beim Einsetzen der heutigen großen Kirchenfenster zerstört hatte, so daß sie sich nur in geringer Tiefe aufdecken ließ. Die auf der Mittelachse der Gebäudefassade platzierte Fensteröffnung im zweiten Obergeschoß kam wieder nahezu unversehrt zum Vorschein und konnte daher fast in ihrer ganzen Tiefe freigelegt werden (Abb. 3). Durch die Freilegung der Fenster wurde eine eindeutige Fassadenrekonstruktion der Kibla-Mauer möglich (Abb. 4), derzufolge die Fenster in drei Stockwerken angeordnet waren und ursprünglich der Einteilung 2-2-1 folgten. Dieser Einteilung dürfte man bei den Fenstern der drei anderen Hauptmauern der Dschami wohl ebenfalls gefolgt sein, obgleich auch teilweise abweichende Lösungsmöglichkeiten bekannt sind. Eine solche Fensteranordnung hatte unter den heimischen Dschamis mit Viereckgrundriß die Dschami des Malkotsch Bej von Siklós ${ }^{29}$ bzw. die 1550/51 erbaute Aladža-Dschami in Foča, ${ }^{30}$ Vakuf des Hasan Nasir (Hasan bin Yusuf), der bis zu seinem Tode das Amt des Ofner Defterdars bekleidete. Baumeister der Dschami ist, nach Ewlija Tschelebi, Ramazan Aga gewesen. ${ }^{31}$ Dieselbe Lösung findet man aber auch bei der Dschami des Hadschi Mehmed Bej (Karadjoz Bej) von Mostar, die 1557 errichtet wurde, ${ }^{32}$ sowie der 1567/68 erbauten »Begluk«- oder »Glavica«-Dschami in Livno, einer Stiftung des zweimal als Ofner Pascha amtierenden Lala Mehmed Paschas. ${ }^{33}$

Der Rekonstruktion der Fassade schlossen sich im Kircheninneren Bauforschungen an der Kibla-Mauer an, in erster Linie um die Überreste der Mihrab aufzudecken. Bei dieser Gelegenheit wurde auch das Fenster im Erdgeschoß vollständig freigelegt, dessen Steinrahmen unversehrt und in situ erhalten blieb. Mit der anschließenden Grabung, für die man parallel zur Mauer einen $50 \mathrm{~cm}$ breiten Suchgraben anlegte, sollte das innere Fußbodenniveau der Dschami bestimmt, ferner der Rest des mutmaßlich unter dem gegenwärtigen Mihrab-Niveau erhaltenen Sockels und das Fundament der Kanzel freigelegt werden (Abb. 5). Im weiteren werde ich die einzelnen Details vor allem in Hinsicht auf die Zusammenhänge behandeln, ohne Unterschied, ob es sich um Überreste der Außenfront oder des Innenraumes handelt.

Die Stärke der Kibla-Mauer, der südöstlichen Hauptmauer, betrug insgesamt $105 \mathrm{~cm}$, das im Erdgeschoß freigelegte Fenster war 118,5 cm breit (Abb. 6). Auf beiden Seiten säumte die Fensteröffnung ein $24 \mathrm{~cm}$ breiter, durch einfache Abkantung gegliederter Steinrahmen, welcher im Inneren unversehrt zum Vorschein kam, während auf Seiten der Fassade ein Teil der Rahmensteine beim Abriß der Vermauerung herausgebrochen wurde. In einem der größeren Stücke waren Löcher, die zum Einsetzen des an der äußeren Front befindlichen Fenstergitters dienten. Mit ihrer Hilfe

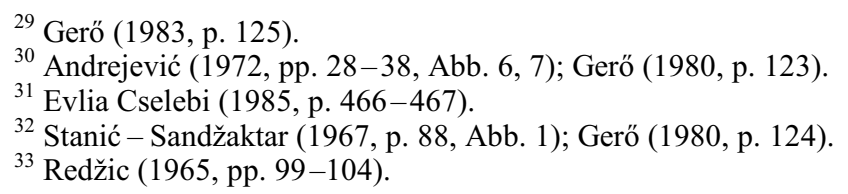




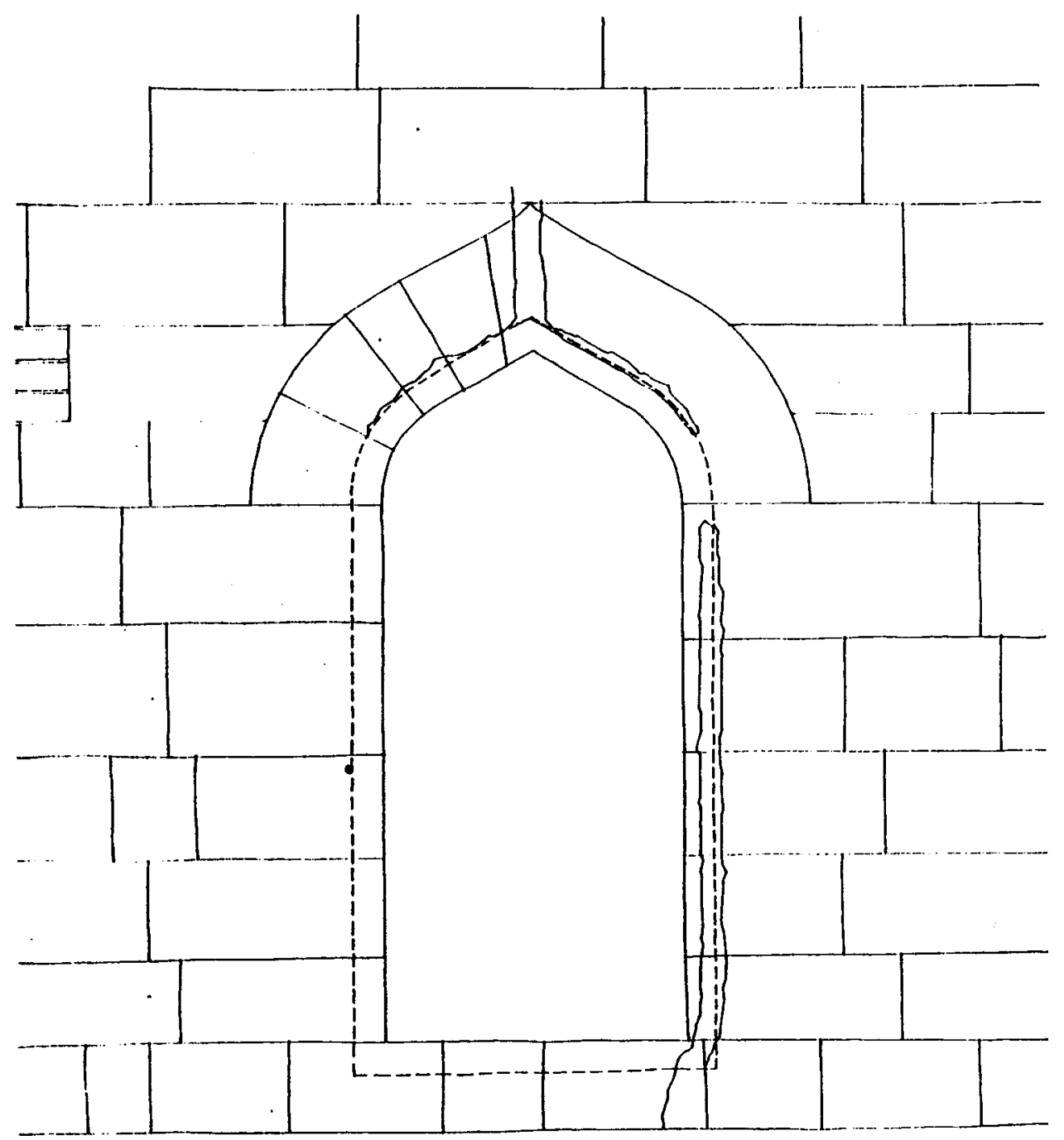

Abb. 3. Das im zweiten Obergeschoß der Westfassade freigelegte türkische Fenster (Dezső Várnai)

kann man die ursprüngliche Einteilung des Gitters mit Knotenbindung rekapitulieren. An einem der Fenster im Erdgeschoß der zur Donau blickenden Front des Hauses Corvin-Platz 3 wurde sekundär ein türkisches Fenstergitter eingebaut, das höchstwahrscheinlich zu einem Erdgeschoßfenster der Dschami Tojgun Paschas gehörte. Die Innenabmessung der Fensteröffnung beträgt $105 \times 201 \mathrm{~cm}$, Verputz und Kalkanstrich ihrer Laibung blieben in gutem Zustand erhalten. Über der gerade abschließenden Fensteröffnung ist eine Oberlichtöffnung mit Kielbogen, die ein aus durchschnittlich 31-35 cm breiten Bogensteinen gestaltetes, vertieftes Spiegelfeld umrahmt. Den Schlußstein des letzteren schmückte an der Frontseite ursprünglich eine Rosette oder 


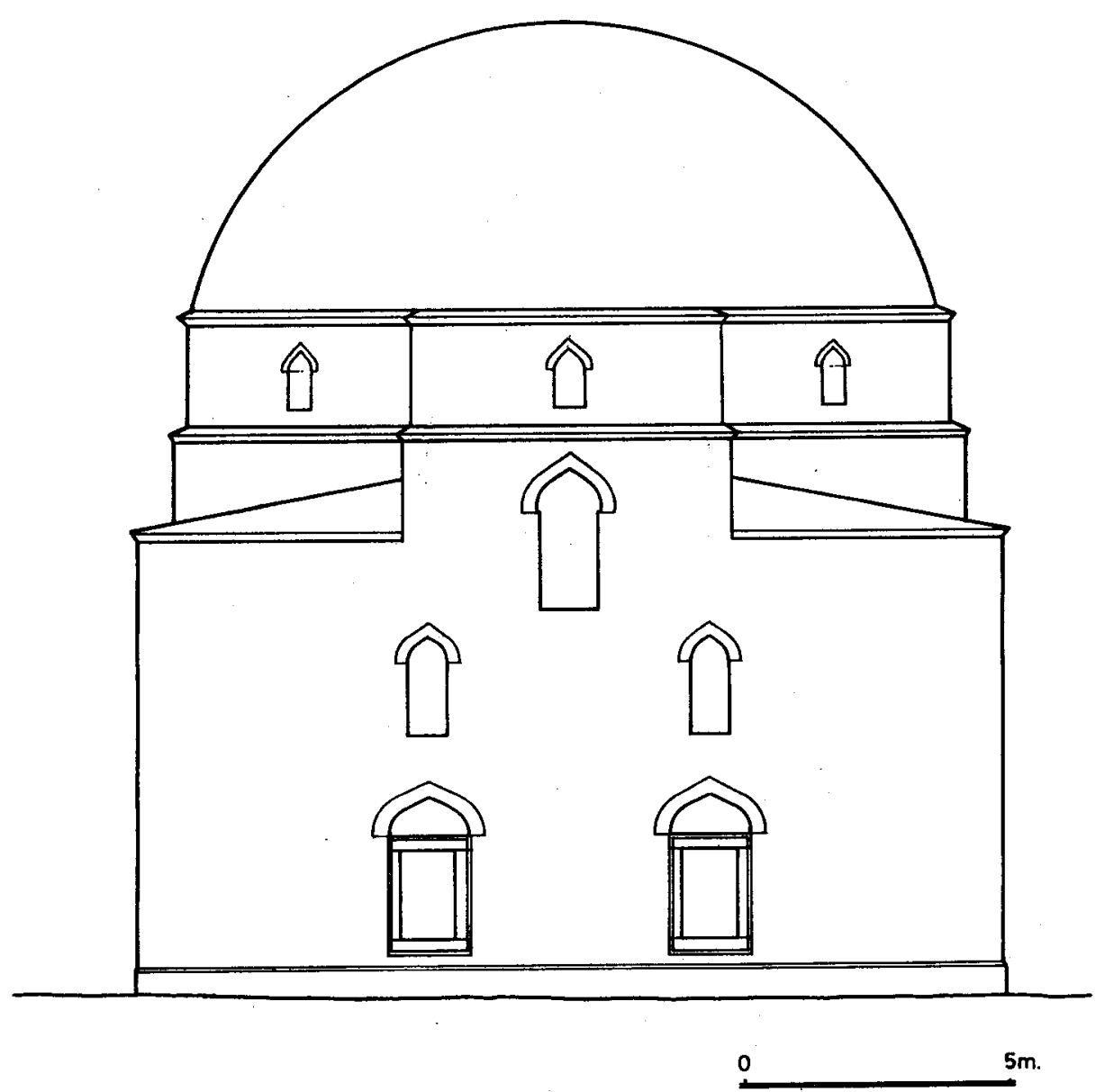

Abb. 4. Rekonstruktion der Südostfassade der Dschami (Kálmán Szíjártó)

Windrose, denn die Spur des Zurückarbeitens dieser Verzierung ist noch heute erkennbar.

Die im ersten und zweiten Obergeschoß befindlichen Fensteröffnungen, ohne Steinrahmung, schlossen ursprünglich mit einem Kielbogen ab (Abb. 3), den wie im obigen Fall ein in Schulterhöhe beginnendes, $34 \mathrm{~cm}$ breites, vertieftes Spiegelfeld säumte. Beide Fenster waren $96 \mathrm{~cm}$ breit, ihre Schulterhöhe betrug $144 \mathrm{~cm}$ und ihre Gesamthöhe bis zur oberen Spitze des Bogens $222 \mathrm{~cm}$. Eine 8-9 cm messende, umlaufende Fuge an der Fassadenfront der Fensteröffnung diente zum Einsetzen des Fensters.

Das zweifellos größte Ergebnis der Forschungen war die Freilegung eines beträchtlichen Teils der Mihrab. Nachdem man den Verputz von dem östlich des türkischen Fensters im Erdgeschoß bis zum barocken Mauerpfeiler der Kirche reichenden Mauerabschnitt entfernt hatte, kamen die Überreste der zugemauerten Mihrab-Nische 


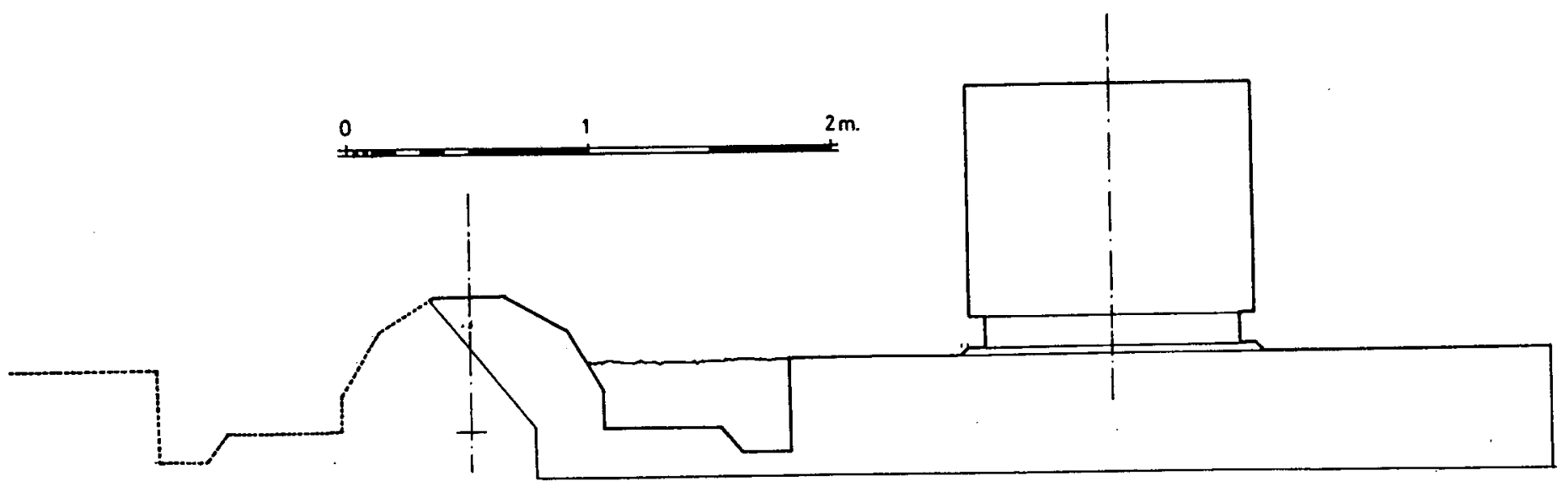

Abb. 5. Grundrißdetail der Kibla-Mauer mit Erdgeschoßfenster und Mihrab (Kálmán Szíjártó) 

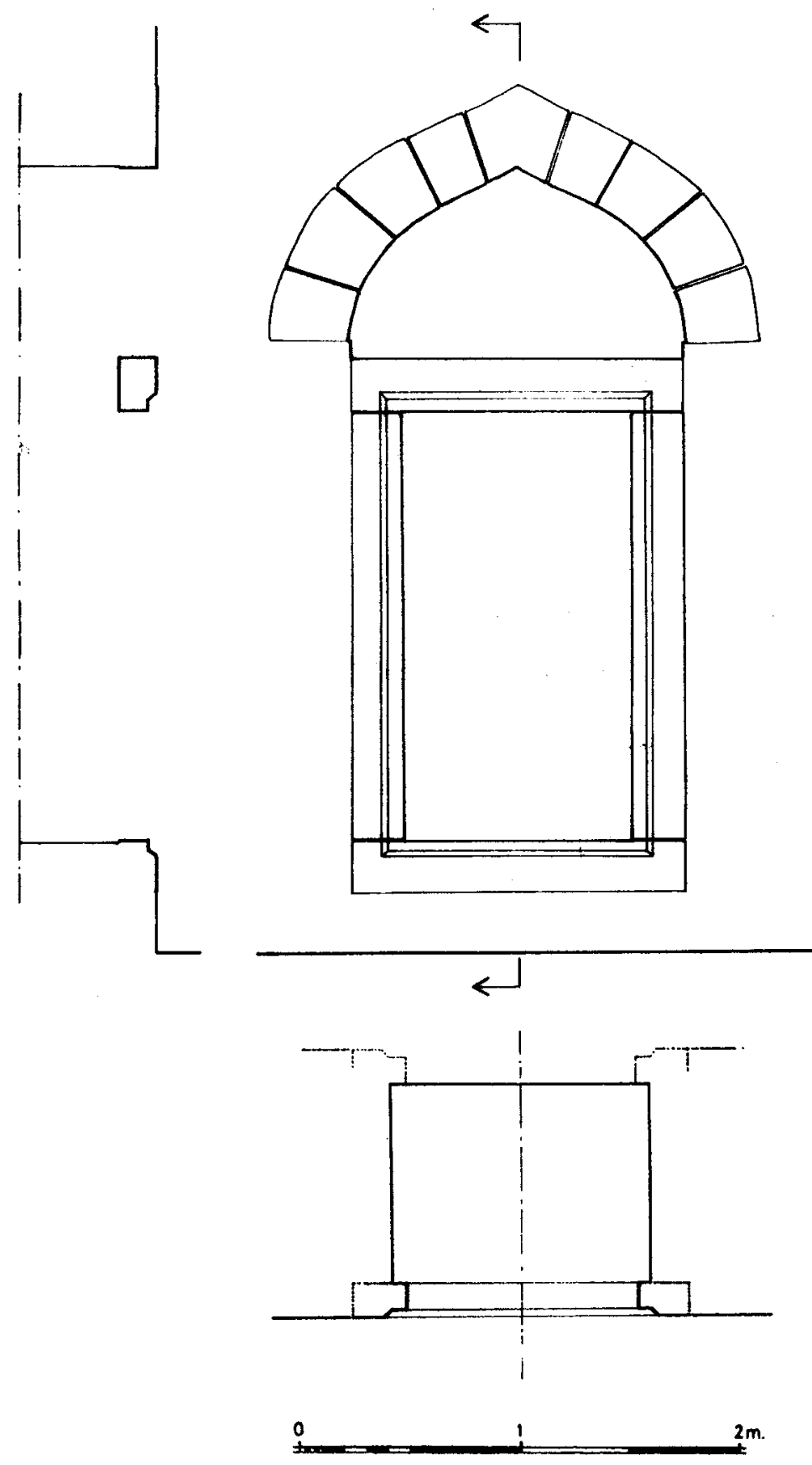

Abb. 6. Das Fenster im Erdgeschoß der Dschami, Grundrißschnitt und Innenansicht (Kálmán Szíjártó) 
und ihres eingemeißelten Rahmens zutage. Es gelang, die Vermauerung abzureißen und anschließend ein größeres Detail des relativ gut erhaltenen mehrstufigen Stalaktitgewölbes auch hinter dem Mauerpfeiler freizulegen. Der unversehrte Sockel des Rahmens befand sich unter dem heutigen Fußbodenniveau. Die zum Vorschein gelangten Details dienten mit wertvollen Angaben, welche eine authentische hypothetische Rekonstruktion der Mihrab ermöglichten (Abb. 7).

Die Mihrab der Dschami Tojgun Paschas ist, ebenso wie die übrigen Reste der Dschami, nicht nur ein herausragendes Denkmal der ungarländischen türkischen Baukunst, sondern besitzt auch vom Gesichtspunkt der Forschung der türkischen Architekturgeschichte in Ungarn in mehrerlei Hinsicht große Bedeutung.

Betrachtet man die Denkmäler der ungarländischen Dschami-Baukunst, kann man feststellen: Schon die Zahl der Dschamis in Ungarn ist gering, und was die Mih$r a b$ anbelangt, so sind es noch weniger. Die hier behandelte Mihrab war voll und ganz in Stein gemeißelt, ihr Gewölbe kann auf Grund der erhalten gebliebenen Details zu den ausgezeichnetsten Werken der ungarländischen türkischen Steinmetzkunst gerechnet werden. Außer ihr gibt es von dieser Art leider nur noch die Mihrab der Dschami des Gazi Kasim Pascha in Pécs, ${ }^{34}$ deren Originaldetails jedoch infolge weitgehender Ergänzungen bei ihrer Restaurierung heute nicht mehr zu bestimmen sind. Von diesen beiden abgesehen entstanden die ungarländischen Mihrab aus Ziegeln und ihr Stalaktitgewölbe aus der auf das Reckteck aufgetragenen Stuckatur.

Eine Seitenplatte der mit sieben Seiten eines Vierzehnecks abschließenden Gebetnische ist jeweils $29,5 \mathrm{~cm}$ breit, während die beiden schmaleren Seiten je $15 \mathrm{~cm}$ messen. Die Nische umgibt ein Steinrahmen aus Quadern, der um $37 \mathrm{~cm}$ aus der Mauerflucht hervorspringt und 76,5 cm, also eine Bauelle, breit ist. Den Rahmen gliedert einfache, $10,4 \mathrm{~cm}$ breite Abkantung, wodurch der äußere Rand nur eine Breite von 19,5 cm erreicht. In $176 \mathrm{~cm}$ Höhe vom ursprünglichen Niveau teilt die Nische eine schmale Schulter, über der die Mauer etwa 1,5 cm aus der Mauerflucht der Nische hervorspringt. Hier fängt das fünfstufige Stalaktitgewölbe an, dessen Elemente je nach Stufe variieren. Die auf Grund der freigelegten Details und der Beobachtungen rekonstruierte Gesamthöhe der Mihrab beträgt $5 \mathrm{~m}$, ihre Breite 2,44 m; die Nische selbst ist 2,20 $\mathrm{m}$ hoch und $1,10 \mathrm{~m}$ breit.

Analogien zur Mihrab unserer Dschami gibt es im heimischen Nachlaßmaterial leider nicht, höchstens Ähnlichkeiten bei einzelnen kleineren Details sind zu entdecken. Nach den Analogien muß man also in erster Linie im Kreis der türkischen Denkmäler des Balkans suchen. Wie schon im Falle der Dschami die Aladža-Dscha$m i$ von Foča als eine der zeitlich nächstliegenden Parallelen zu betrachten ist - deren architektonische Details jedoch eine wesentlich reichere Gestaltung aufweisen -, so gilt das auch für ihre Mihrab. Beide besitzen ein fünfstufiges, in Stein gehauenes Stalaktitgewölbe, deren Elemente zum Großteil identisch oder zumindest sehr ähnlich sind. Auch in ihren Abmessungen stimmen sie annähernd überein, da die Mihrab der Aladža-Dschami ohne den reichen Giebelschmuck ebenfalls etwa $5 \mathrm{~m}$ hoch und zweiunddreiviertel Meter breit ist.

${ }^{34}$ Gosztonyi (o.J. p. 34).

Acta Orient. Hung. 54, 2001 


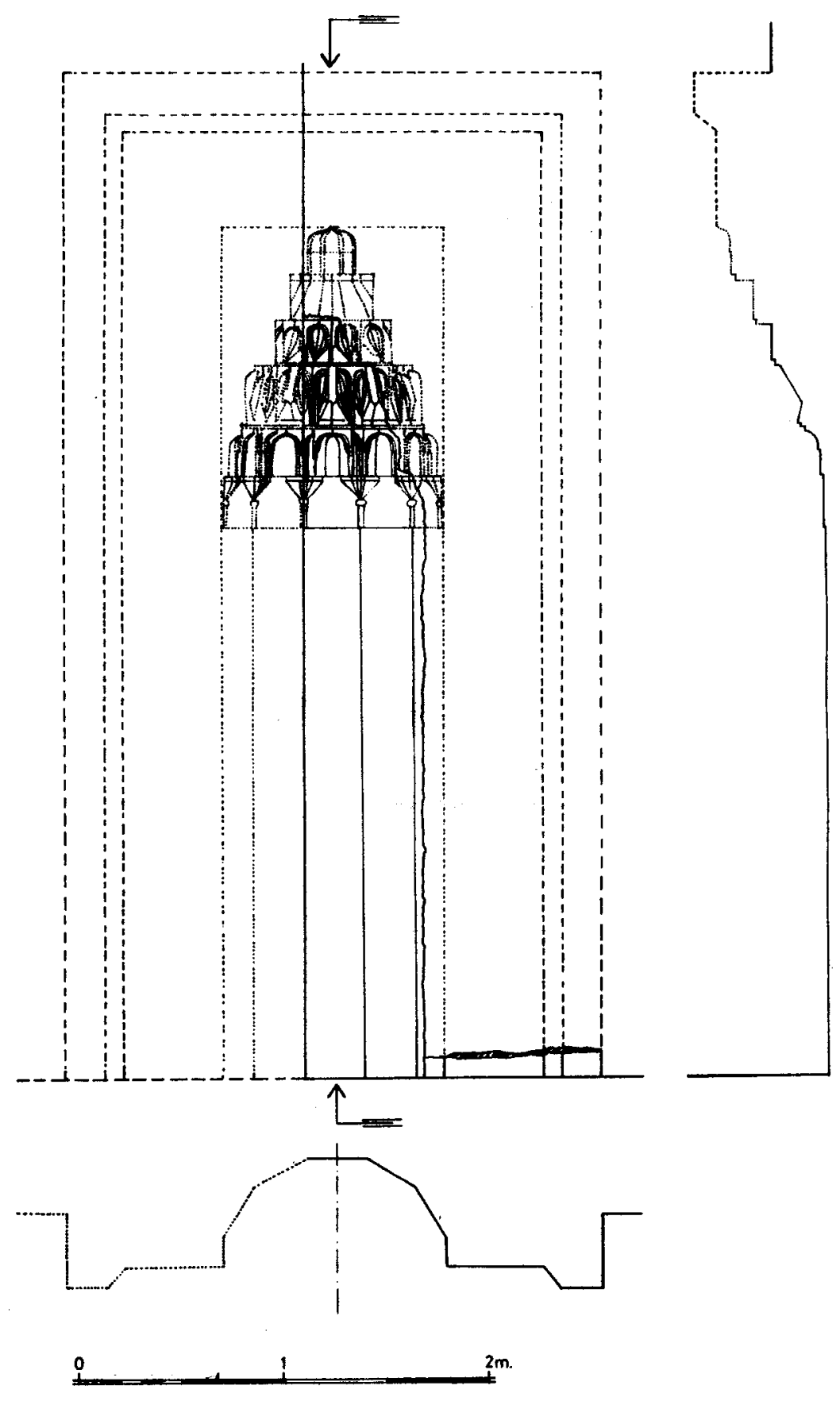

Abb. 7. Rekonstruktion der Mihrab mit Kennzeichnung der erhaltenen Originaldetails, Grundrißschnitt und Ansicht (Kálmán Szíjártó) 
Etwas rechts vom Fenster neben der Mihrab kam ein Rest des in Stein gemeiBelten Mimber-Fundaments zum Vorschein.

Die Ergebnisse der Freilegung der Dschami Tojgun Paschas im Stadtteil Buda-Víziváros lassen sich wie folgt zusammenfassen: Die Dschami war ursprünglich ein Bau mit Viereckgrundriß, einer Vorhalle mit drei Gewölbeabschnitten und mit einem $\mathrm{Mi}$ narett (Abb. 8). Von ihrer südöstlichen Hauptmauer und deren architektonischen Elementen blieben in der Fassade und im Inneren der jetzigen Kapuzinerkirche bedeutende Details erhalten. Die originale 2-2-1-Fensteranordnung des würfelförmigen Zentralbaus konnte ebenso rekonstruiert werden wie die in Stein gemeißelte Mihrab, deren feingearbeitetes Stalaktitgewölbe eines der schönsten Denkmäler türkischer Baukunst dieser Art in Ungarn ist. Die erhalten gebliebenen Details sowie die Analogien, Stadtpläne und Ansichten haben nicht nur die Rekonstruktion ihres Grundrisses, sondern auch eine hypothetische Rekonstruktion der Dschami selbst ermöglicht.

Als erste unter den Stiftungseinrichtungen des Tojgun Pascha dürfte die das religiöse Zentrum des Mahalle bildende Dschami entstanden sein, deren Bau man in die erste Amtszeit Tojguns als Pascha von Ofen setzen kann. Um so mehr, als Dernschwam anläßlich seines Ofner Besuchs im August 1555 über die Bauarbeiten am Bad berichtet. Demnach kann man die Bauzeit der Dschami in den Zeitraum 15531555 datieren.

Das zweite Vakuf-Gebäude Tojgun Paschas markiert die Karte von de la Vigne südlich der Dschami. Zwischen beiden Gebäuden verläuft ein schmaler Korridor. Dieser Bau war - nach den Beobachtungen der kleineren Grabung - ursprünglich das Gebäude des Kapuzinerklosters und stand im Gebiet des Klosterhofs. Die Grabung bot nur Möglichkeiten zur topographischen Identifizierung, denn an Hand der zum Vorschein gelangten Details - sekundär in der hier aufgefundenen Mauer des Bades verbaute gotische Architekturfragmente -, konnten die Zusammenhänge nicht geklärt werden. Allerdings bestätigten sie Dernschwams Bericht. Diese und andere skulptierte Steine stammten höchstwahrscheinlich vom Abriß der nahegelegenen mittelalterlichen Kirche des Augustinerordens oder dessen Kloster.

Das Bad erwähnt außer Dernschwam nur Ewlija Tschelebi, und auch Marsigli führt es in seinen Erinnerungen an die Rückeroberung des Jahres 1686 an. Im August 1555 war das Bad schon im Bau. Wann es jedoch beendet wurde, kann nur vermutet werden, da man nicht weiß, in welchem Stadium es sich zum genannten Zeitpunkt befand. Möglicherweise wurden die Bauarbeiten bis zum Februar 1556 abgeschlossen, aber auch daß sie im Jahre 1558 weitergingen, ist nicht auszuschließen. Marsigli bezeichnet das Bad als Hamam, was Doppelbad bedeutet. ${ }^{35}$

${ }^{35}$ Hamam bedeutet im Arabischen und im Türkischen allgemein Bad. Zur Unterscheidung versteht der Türke darunter in erster Linie Dampfbad, das Thermalbad nennt er Ilidsche oder Kaplidscha. Eine Ausnahme bildet das Doppelbad - Tschifte Hamam; in diesem Fall bezieht sich der Name auf beide Typen des Bades.

Acta Orient. Hung. 54, 2001 


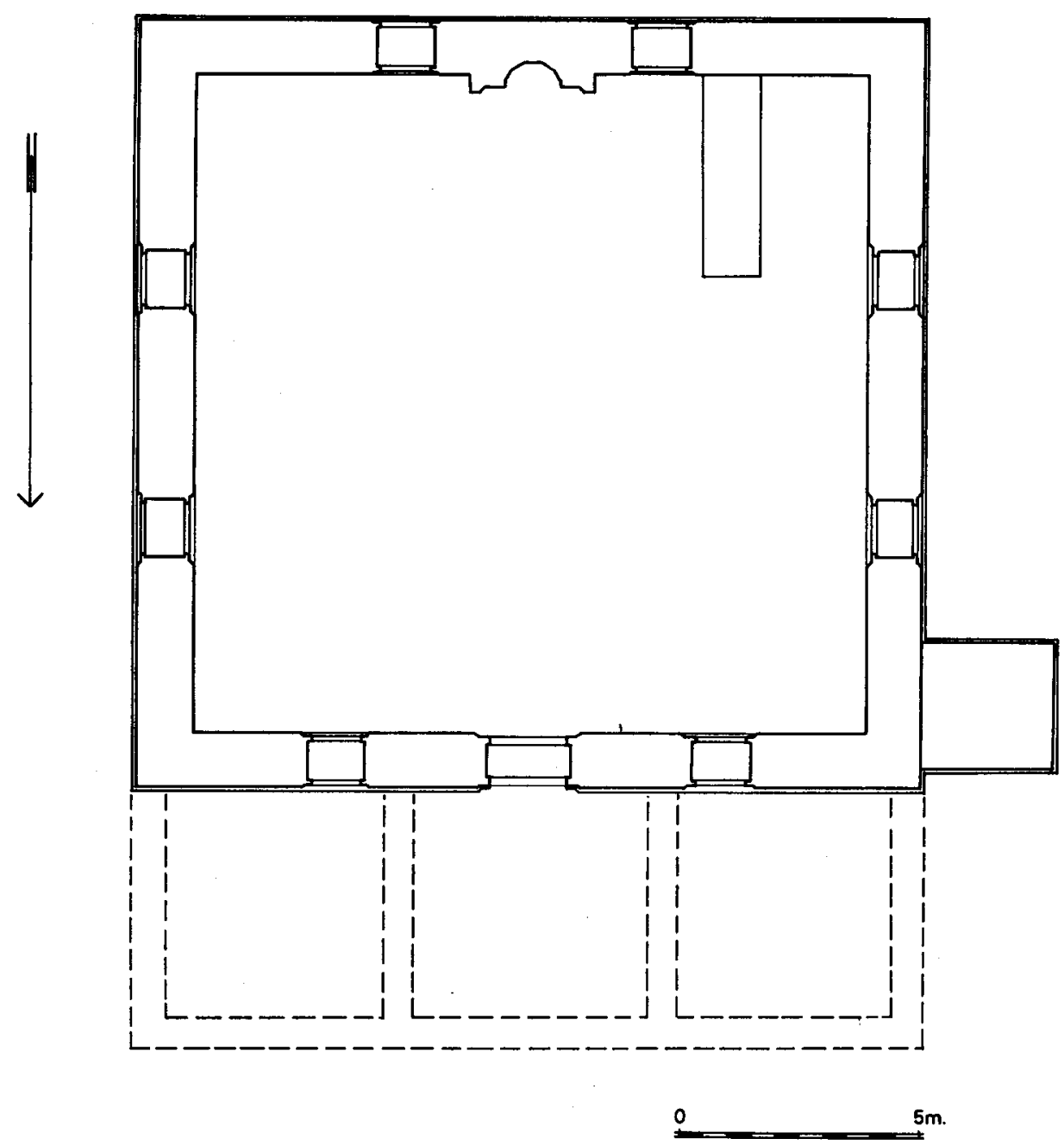

Abb. 8. Rekonstruierter Grundriß der Dschami (Kálmán Szíjártó)

\section{Literatur}

Andrejević, A. (1972): Aladža džamija u Foči. In: Filozofski fakultet u Beogradu Institut za Istoriju Umetnosti. Beograd (Monografia 2).

Dernschwam, H. (1923): Hans Dernschwams Tagebuch einer Reise nach Konstantinopel und Kleinasien (1553-1555). Hrsg. Franz Babinger. München-Leipzig.

Dernschwam, H. (1984): Erdély, Besztercebánya, Törökországi útinapló. (Közreadja Tardy Lajos.) Budapest, Európa Könyvkiadó. 
Evlia Cselebi (1985): Evlia Cselebi török világutazó magyarországi utazásai 1660-1664. (Ford.: Karácson Imre. Az előszót és a szómagyarázatot írta, a jegyzeteket átdolgozta: Fodor Pál.) Budapest, Gondolat Kiadó.

Fekete, L. (1944): Budapest a törökkorban. (Budapest története III.) Budapest.

Fekete, L. - Káldy-Nagy, Gy. (1962): Budai török számadáskönyvek 1550-1580. Budapest, Akadémiai Kiadó.

Gerő, Gy. (1980): Az oszmán-török épitészet Magyarországon (Dzsámik, türbék, fürdők). Budapest, Akadémiai Kiadó (Mủvészettörténeti Füzetek 12).

Gerő, Gy (1983): A siklósi Malkocs bej dzsámi. In: Épités-Épitészettudomány XV. Budapest, pp. $117-131$.

Gévay, A. (1841): A' budai pasák. Wien.

Gosztonyi, Gy. (o.J.): A várostemplom épitéstörténete. Pécs.

H. Gyürky, K. (1971): Adatok a budai Szent Péter külváros topográfiájához. In: Budapest Régiségei XXII. Budapest, pp. 235-240.

Redžić, H. (1965): Restauratorskonservatorski nedovi na "Glavici” džamija u Livno. Naše Starine X, pp. 99-104.

Rózsa, Gy. (1963): Budapest régi látképei (1493-1800). Budapest.

Sahm, W. (1912-1920): Beschreibung der Reisen des Reinhold Lubenau. Mitteilungen aus der Stadtbibliothek zu Königsberg i. Pr. IV. Königsberg.

Stanič, R. - Sandžaktar, M. (1967): Konservacija Karadjoz begove medrese u Mostaru. Naše Starine $\mathrm{XI}$.

Velics, A. - Kammerer, E. (1890): Magyarországi török kincstári defterek, II. Budapest.

Veress, E. (1906): Gróf Marsigli Alajos Ferdinánd olasz hadi mérnök jelentései és térképei Budavár 1684-1686-iki ostromairól, visszafoglalásáról és helyrajzáról. Budapest Régiségei IX, pp. $103-170$.

Végh, A. (1998): A középkori ágostonos kolostor felfedezése a Vízivárosban. Magyar Múzeumok 1998/3. Vol. 4, pp. 15-17.

Veremund, P. (1927): Emlékkönyv a budai kapucinusrendi zárda és plébániatemplom renoválásának alkalmából. Budapest, 80 pages. 